\title{
OPEN Polycrystalline Ni nanotubes under compression: a molecular dynamics study
}

\author{
J. Rojas-Nunez ${ }^{1,2}$, S. E. Baltazar ${ }^{1,2}$, R. I. Gonzalez ${ }^{2,3}$, E. M. Bringa ${ }^{3,4}$, S. Allende ${ }^{1,2}$, M. Kiwi' ${ }^{2,6} \&$ \\ F. J. Valencia ${ }^{2,5 \bowtie}$
}

Mechanical properties of nanomaterials, such as nanowires and nanotubes, are an important feature for the design of novel electromechanical nano-architectures. Since grain boundary structures and surface modifications can be used as a route to modify nanostructured materials, it is of interest to understand how they affect material strength and plasticity. We report large-scale atomistic simulations to determine the mechanical response of nickel nanowires and nanotubes subject to uniaxial compression. Our results suggest that the incorporation of nanocrystalline structure allows completely flexible deformation, in sharp contrast with single crystals. While crystalline structures at high compression are dominated by dislocation pinning and the multiplication of highly localized shear regions, in nanocrystalline systems the dislocation distribution is significantly more homogeneous. Therefore, for large compressions (large strains) coiling instead of bulging is the dominant deformation mode. Additionally, it is observed that nanotubes with only $70 \%$ of the nanowire mass but of the same diameter, exhibit similar mechanical behavior up to 0.3 strain. Our results are useful for the design of new flexible and light-weight metamaterials, when highly deformable struts are required.

The development of lightweight hierarchical metallic nanostructures constitutes an alternative to create materials with novel and unexpected mechanical properties, a subject that recently has attracted much attention and has been extensively studied ${ }^{1,2}$. Usually, these structures correspond to a collection of mostly nanocrystalline (nc) nanotubes (NTs) or nanowires (NWs), self-assembled as building blocks and suitably arranged to ensure, for a given deformation, a cooperative response of all constituents $\mathrm{s}^{3-6}$. Thus, the mechanical characteristics are dependent on the nanostructure design, and on the properties of the nano-objects used as building blocks ${ }^{7}$. Extensive research has been conducted on the mechanical properties of metallic crystalline (c-NWs) and nanocrystalline nanowires (nc-NWs) ${ }^{8-13}$. However, the hollow counterpart has not received much attention, probably due to the lack of experimental data on these kind of materials. Nowadays, the development of atomic layer deposition techniques and the Kirkendall effect allow the successful synthesis of nanocrystalline nanotubes with an adjustable combination of geometrical parameters ${ }^{14-16}$, and a nanocrystalline texture close to $5 \mathrm{~nm}$ grain size ${ }^{17}$. It is worth noticing that NTs are a lightweight version of NWs, but with a larger surface to volume ratio and smaller specific density, features that make them excellent candidates for their use in catalysis, energy devices, and the design of novel nanostructures ${ }^{18}$. The use of NTs and NWs for nanostructured devices requires an understanding of how the filaments respond under tensile and compressive deformation. Molecular dynamics simulations have shown that single crystal NT mechanical properties can be tailored by adjusting both inner and outer surfaces ${ }^{19-21}$.

However, simulations of nc-NTs have not received the same attention as simulations of c-NTs, even though they are an excellent approximation to the typically obtained experimental conditions ${ }^{16}$. A detailed study of $\mathrm{Ni}$ nc-NTs under tension was carried out by Rojas-Nunez et al. ${ }^{17}$ revealing that nc-NTs in the elastic regime, with similar yield strength and a mass reduction close to $60 \%$, behave similarly to nc-NWs. They have just a slightly different fracture point. Finite element models under compression computed buckling of thin-walled NTs, but the model did not include complex defects, such as dislocations or grain boundaries ${ }^{22}$. On the other hand, molecular dynamics simulations show that thin crystalline face centered cubic (fcc) NWs deform by stacking

\footnotetext{
${ }^{1}$ Departamento de Física, Universidad de Santiago de Chile, USACH, Av. Ecuador, 3493 Santiago, Chile. ${ }^{2}$ CEDENNA, Universidad de Santiago de Chile, USACH, Av. Ecuador, 3493 Santiago, Chile. ${ }^{3}$ Centro de Nanotecnología Aplicada, Facultad de Ciencias, Universidad Mayor, Providencia, Chile. ${ }^{4}$ CONICET and Facultad de Ingeniería, Universidad de Mendoza, 5500 Mendoza, Argentina. ${ }^{5}$ Centro de Investigación DAiTA Lab, Facultad de Estudios Interdisciplinarios, Universidad Mayor, Santiago, Chile. ${ }^{6}$ Departamento de Física, Facultad de Ciencias, Universidad de Chile, Santiago, Chile. ${ }^{\varpi}$ email: felipe.valenciad@gmail.com
} 


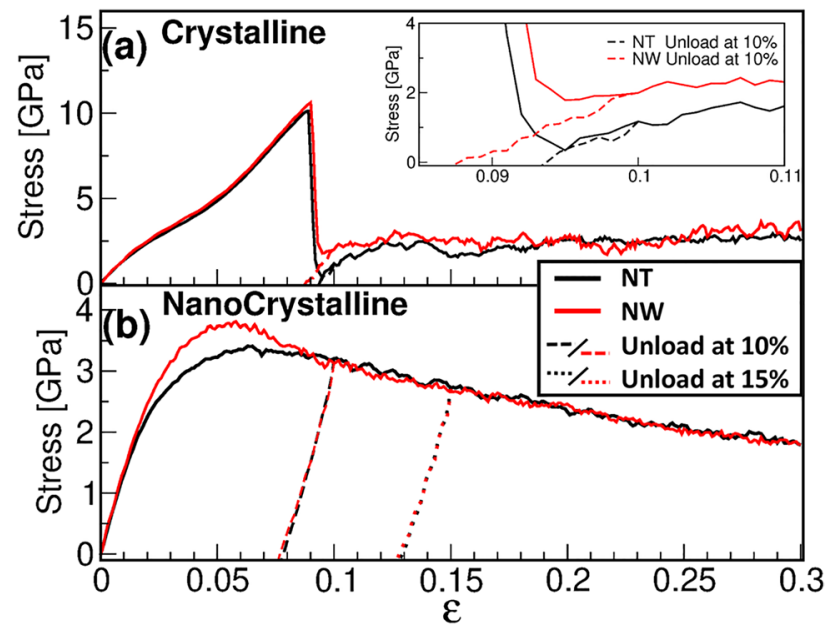

Figure 1. Compression stress-strain plots for (a) crystalline NT and NW with 22nm external diameter; (b) nanocrystalline NT and NW with $22 \mathrm{~nm}$ external diameter and $5 \mathrm{~nm}$ wall thickness. Dashed lines correspond to recovery during unloading to zero stress.

faults and twins driven by surface tensile stress, assisted by partial dislocations ${ }^{10}$. Monk and Farkas ${ }^{23}$ showed the asymmetric behavior under tension-compression of Ni nc-NWs of $10 \mathrm{~nm}$ grain size, and concluded that plasticity in relatively small diameter NWs is mediated by grain boundary sliding, rather than by dislocation activity. Modeling nc-NWs and nc-NTs under experimental conditions implies grain sizes where grain boundary activity cannot be safely ignored. Additionally, the large specific surface areas of NTs allows a richer behavior of grain boundary and dislocation motion. Both factors could lead to materials with unexpected mechanical properties. To treat all these issues we implemented molecular dynamics simulations to investigate the effect of mechanical compression on nanocrystalline and crystalline NTs and NWs. We studied the dominant mechanisms in the plastic regime for large compressive deformations, and compared the difference between nc-NTs and nc-NWs with the respective pristine counterparts. In addition, the way these systems recover from different deformation scenarios is discussed.

\section{Results}

Mechanical compression of Ni nanotubes and nanowires. To compare the plasticity mechanisms of crystalline and nanocrystalline materials we carried out a comparison of the same diameter (22 nm) nanotubes and nanowires. The aspect ratio, defined as the ratio between NT length and thickness, was set to 4.8 for all samples. The relevance of the aspect ratio in the ductile to brittle fracture of nanocrystalline nanowires was discussed by $\mathrm{Wu}$ et al. $^{24}$; we adopted the aspect ratio of 4.8 to reduce possible finite size effects due to the characteristic length scale of the plastic deformation sources, such as dislocations, and fracture or shear localization zones ${ }^{25}$.

Uniaxial compression is applied to both crystalline and nanocrystalline NTs and NWs until a strain of 0.3 is reached. Fig. la shows the behavior of c-NTs and c-NWs, whose characteristic elastic regime extends up to strains of 0.09 , and includes non-linear elasticity. The plasticity starts with a significant stress drop right after the ultimate strength for both structures is achieved, with a stress reduction of nearly $97 \%$ for NTs, and $84 \%$ for NWs. This abrupt fall is due to multiple slips in the preferential planes ${ }^{23}$ along the NT (and NW) diameter ${ }^{10}$, assisted by partial dislocations across the NT/NW that leave stacking faults behind. The DXA algorithm from OVITO provides identification of different types of dislocations, including fcc crystals full, Shockley, stair-rod, Hirth, and Frank dislocations. OVITO does not provide information on dislocation junctions, but stair-rod, Hirth, and Frank dislocations are sessile dislocations which typically appear as the result of dislocation junctions ${ }^{26}$. The c-NW stress drop for $\epsilon=0.1$ is caused by the nucleation and propagation of partial dislocations, usually associated with softening. However, the large number of partials rapidly leads to multiple dislocation junctions, identified by OVITO as a large number of sessile stair-rod dislocations which lead to hardening. Both effects produce a nearly constant stress flow at $2.5 \mathrm{GPa}$, for NWs and NTs. Fig. S1 illustrates this, showing the dislocation network for a c-NW.

On the other hand, both NTs and NWs with nanocrystalline structures show a completely different macroscopic behavior. Figure $1 \mathrm{~b}$ reveals a smooth transition from elastic to plastic regimes, as expected from previous nc simulations ${ }^{17}$. At the nanoscale, metallic single crystal deformation simulations usually display a linear regime for small strain, even for single crystals with dislocations ${ }^{27}$. However, for larger strains, a non-linear elastic regime can precede dislocation activity due to the anharmonic interaction potential. Poly-crystalline simulations typically show non-linearities even at very small strain before dislocation activity starts due to grain boundary activity ${ }^{28}$.

The ultimate stress is reached at compressions of nearly 0.05 , and the elastic limit is estimated to be a 0.02 strain for both nc-NTs and nc-NWs, on the basis of the loading and unloading plots. For all the cases studied, the NT and/or NW shapes do not significantly affect mechanical properties. This is because the grain boundary 
(a)
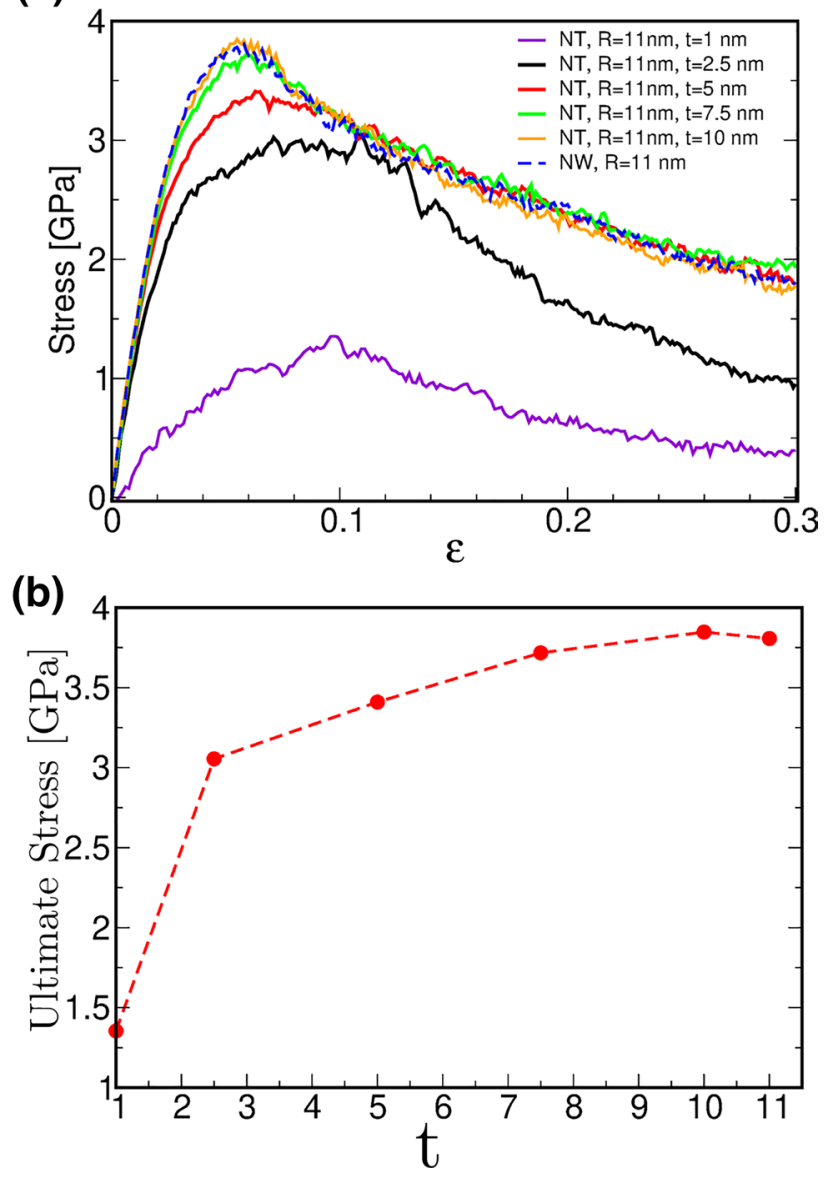

Figure 2. Mechanical response for different nc-NTs. (a) Compression stress-strain plots for different nc-NTs wall thicknesses $(\mathrm{t})$. The blue dashed line corresponds to an $11 \mathrm{~nm}$ radius $\mathrm{nc}-\mathrm{Nw}$. (b) Ultimate stress as function of NT thickness.

\begin{tabular}{|l|l|l|l|l|l|l|}
\hline $\mathbf{t}(\mathbf{n m})$ & $\mathbf{1 . 0}$ & $\mathbf{2 . 5}$ & $\mathbf{5 . 0}$ & $\mathbf{7 . 5}$ & $\mathbf{1 0}$ & $\mathbf{1 1}$ \\
\hline$\% \mathrm{~m}_{n t} / \mathrm{m}_{n w}$ & $17.4 \%$ & $40.3 \%$ & $70.3 \%$ & $89.9 \%$ & $99.1 \%$ & $100 \%$ \\
\hline Ultimate stress $(\mathrm{GPa})$ & 1.35 & 3.05 & 3.41 & 3.71 & 3.84 & 3.80 \\
\hline
\end{tabular}

Table 1. Ultimate stress, and relative mass ratio between nanotube $\left(m_{n t}\right)$ and nanowire $\left(m_{n w}\right)$, for different thickenesses. $\mathrm{t}=11 \mathrm{~nm}$ represents the nanowire case.

structure, in particular the ultrafine grains, seem to control several features, as the yield stress, the elastic limit, and the plastic behavior.

In addition to compressive loading we carried out the unloading of the systems from 0.1 and 0.2 strain. The stress-strain slope during recovery is similar to the loading slope. The residual strain is always larger than 0.07 and 0.17 for strains of 0.1 and 0.2 , respectively. We observe that the crystalline nanotube and nanowire (Fig. 1a) have a larger residual strain than the nc-NTs and nc-NWs (Fig. 1b). Using the CAT tool ${ }^{29}$ it was observed that, for single crystals, more than $90 \%$ of defective atoms belong to twin boundaries, while only some isolated stacking faults remain after the unloading process. The large and non-recoverable twin boundaries, which often cross the whole diameter of the single crystal structure, are consistent with the larger residual strain observed for the single crystals as compared to polycrystals (Fig. S2). Unloading from a relatively small plastic strain does not lead to full de-twinning or recovery of the few existing dislocation junctions. In fact, at the end of the unloading process, the strain that corresponds to an initial 0.1 is 0.093 for c-NTs, 0.087 for c-NWs, and 0.077 for both nc-NWs and nc-NTs.

nc-NTs and nc-NWs show similar mechanical performance. The nc-NTs wall thickness $(\mathrm{t})$ dependence under compression is illustrated in Fig. 2a. As expected, the early plasticity stages are dominated by GB sliding; the thinner the thickness the earlier is the deviation from linear elastic behavior, and the lower the ultimate stress. The minimum shell thickness to obtain the same mechanical behavior than NWs is close to $\mathrm{t}=7 \mathrm{~nm}$, while values 


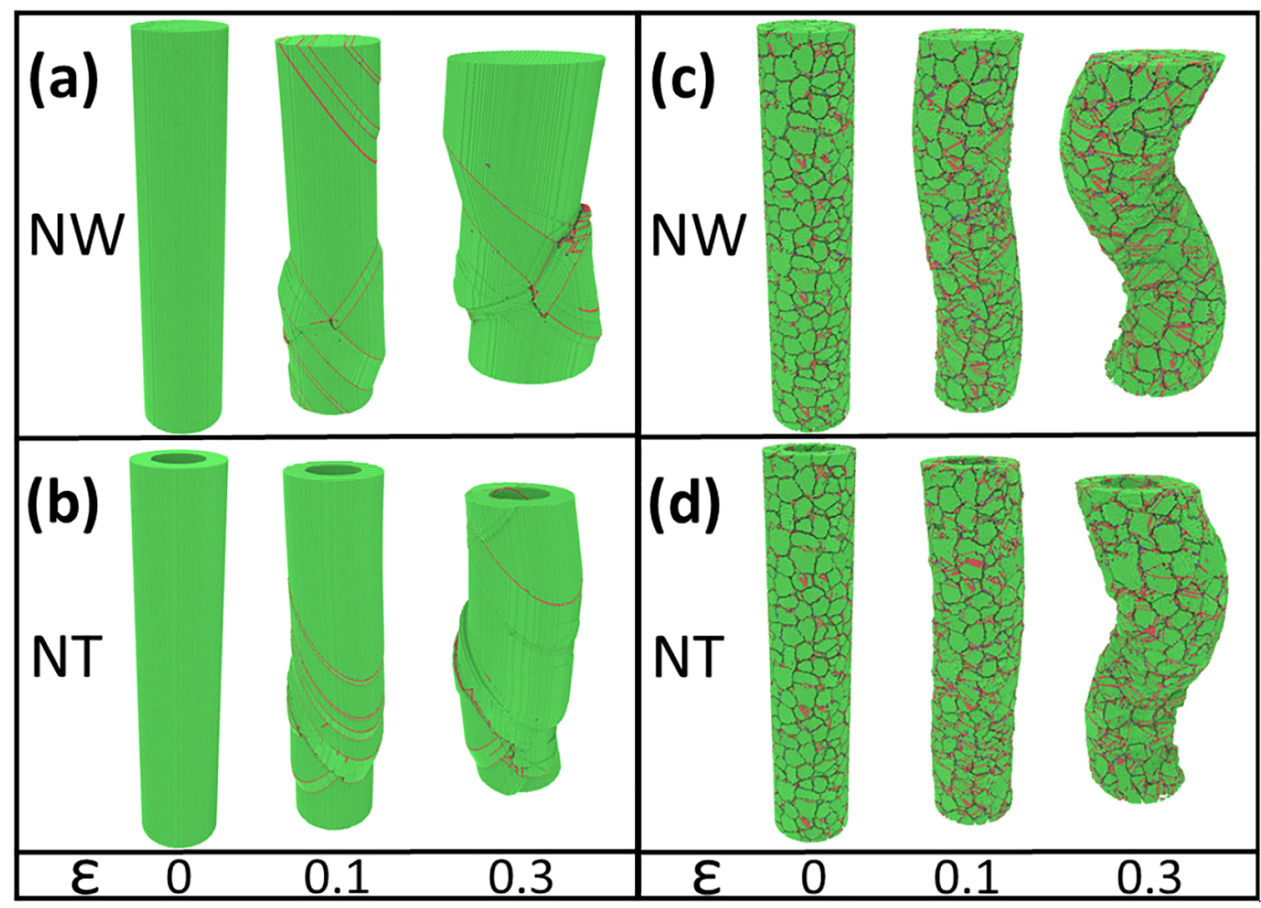

Figure 3. Illustration of NWs and NTs at different strains. (a,b) snapshots of crystalline NWs and NTs under different compression values. (c,d) nanocrystalline NWs and NTs under different compressions. The GB atoms are removed for clarity. The fcc (green), bcc (blue), and hcp (red) atoms were detected by common neighbor analysis (CNA).

smaller than $5 \mathrm{~nm}$ have incidence not only on the maximum strength, but also on material plastic flow even for large deformations (Fig. 2b). For strains larger than 0.1 all curves are similar, except for the thinner thicknesses where the effective grain size is smaller, and surface effects are dominant, enhancing the grain boundary sliding towards the inner surfaces. We note that the hollow structure leads to mass reduction relative to the nanowire case. In Table 1, we summarize the relative mass with respect to nanowire mass for the cases we simulated, and the corresponding ultimate tensile stress, as displayed in Fig. $2 \mathrm{~b}$. We note that $\mathrm{t}=5 \mathrm{~nm}$ represents a critical value, with a NW mass reduction of $30 \%$, but which still has the same Young modulus and flow stress of the heavier $\mathrm{NW}$, for strains larger than 0.1 . In this case, the ultimate stress reduction is of only $0.4 \mathrm{GPa}(\epsilon=0.1)$. For larger mass reduction, mechanical properties are not so well preserved.

As expected, in crystalline samples plasticity is dominated by partial dislocations along $\{111\}$ planes, at $45^{\circ}$ to the loading axial direction, as illustrated in Fig. 3a,b. Several stacking faults, located in consecutive planes, lead to twinning. Stacking faults and twins lead to shear localization, and a noticeable bulging is appreciated for strains larger than 0.1 . However, in nanocrystalline systems the situation is quite different, since the small grain size $(<5 \mathrm{~nm})$ implies that the material can be assumed to be an almost isotropic solid, where dislocation activity also takes place in the $\{111\}$ planes, but with many orientations and dislocations of a characteristic length not larger than the grain size, leading to a buckling deformation mode, instead of the bulging observed in single crystals (Fig. 3c,d). It is known that in some NWs under compression dislocation activity can dominate plasticity ${ }^{23,30,31}$; however, grain boundary sliding cannot be completely ruled out. Figures $3 \mathrm{c}$,d, show that strains of 0.1 lead to an increase in roughness, with a $\Delta r$ value of $0.8 \mathrm{~nm}$ along nc-NT and nc-NW surfaces. For larger strains $(>0.20)$ grain boundary activity leads to buckling, with $\Delta r \geq 3.5 \mathrm{~nm}$ for both nc-NTs and nc-NWs.

In order to follow the grain evolution of nanocrystalline NTs and NWs we used the local crystalline orientation $^{32}$ depicted in Figs. S3, and S4, respectively. At low strains, both NTs and NWs show grains with few defects and almost no rotation. However, for strains larger than 0.1 the coiling shows clear differences, while the buckling wavelength of the NTs and NWs remains almost constant. Thus, we notice that there are still some discrepancies about the validity of continuum buckling theory at the nanoscale ${ }^{33}$. However, if we assume buckling could be described by the Euler-beam theory, then it will be affected by the aspect ratio of the nanotubes ${ }^{34}$.

To investigate the role of the radius we simulate $\mathrm{R}=13$ and $15 \mathrm{~nm} \mathrm{NTs}$ for $\mathrm{t}=2.5 \mathrm{~nm}$ and $5 \mathrm{~nm}$. As is observed in Fig. 4a,b the NT thickness is the most relevant parameter to describe the mechanical behavior. For both radius, the maximum stress is achieved for $5 \mathrm{~nm}$, with a similar flow stress at larger strains, while the weaker response is obtained from $\mathrm{t}=2.5 \mathrm{~nm}$. As it was previously mentioned, the ultra-small wall enhances material migration to the inner surface. The consequence of grain activity, in particular, grain boundary rotation, appears as surface buckling during the coiling process, as seen in the inset of Fig. 4c.

Buckling appears above 0.15 strain and contributes to plasticity. We investigated the tube geometry, shown in Fig. $4 \mathrm{c}$, and find that the inner diameter of the tube reaches values as small as $\sim 2 \mathrm{~nm}$ at the buckling site, which is a reduction of nearly $90 \%$ of the initial diameter. To study in detail this geometry we computed the surface 
(a)

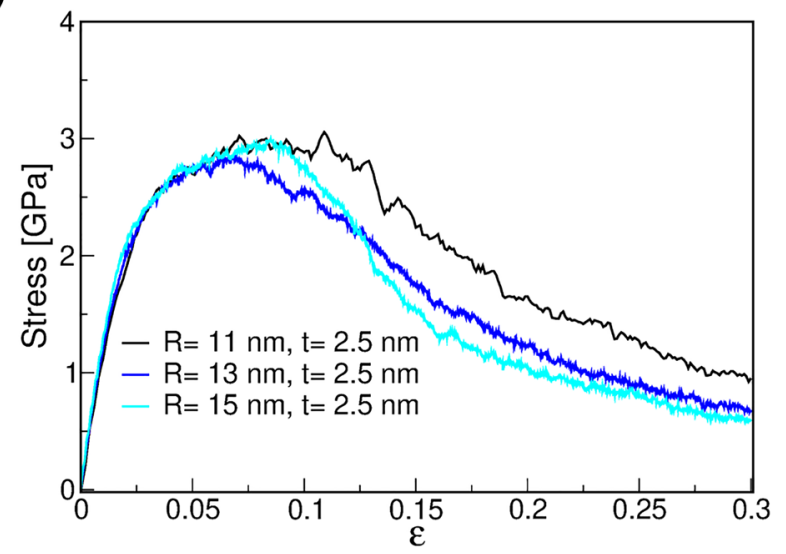

(b)

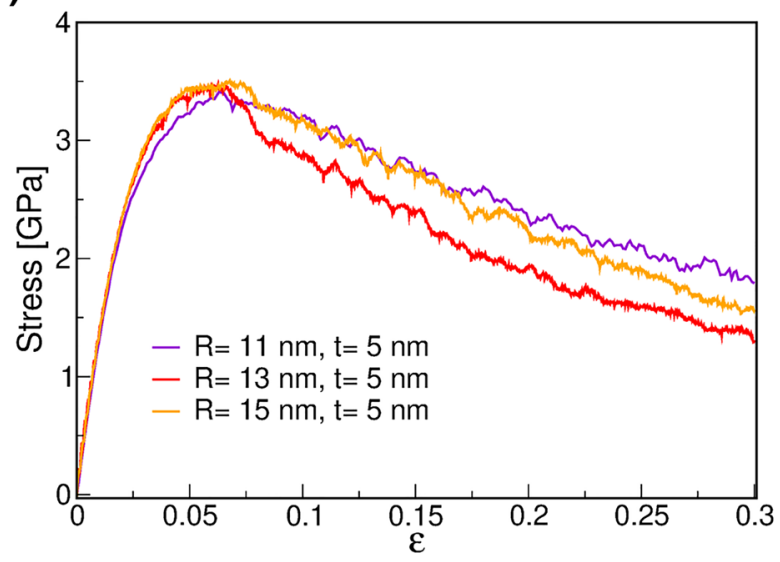

(c)
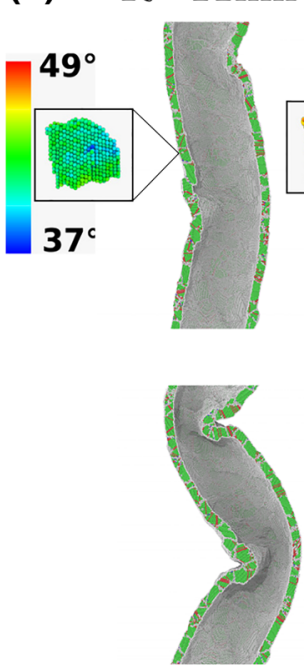

\section{$\mathrm{R}=13 \mathrm{~nm}$}

$\mathrm{R}=15 \mathrm{~nm}$
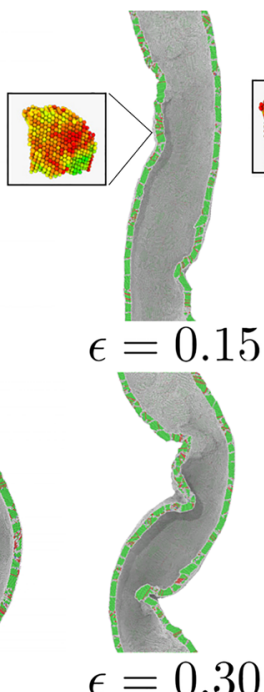

(d)

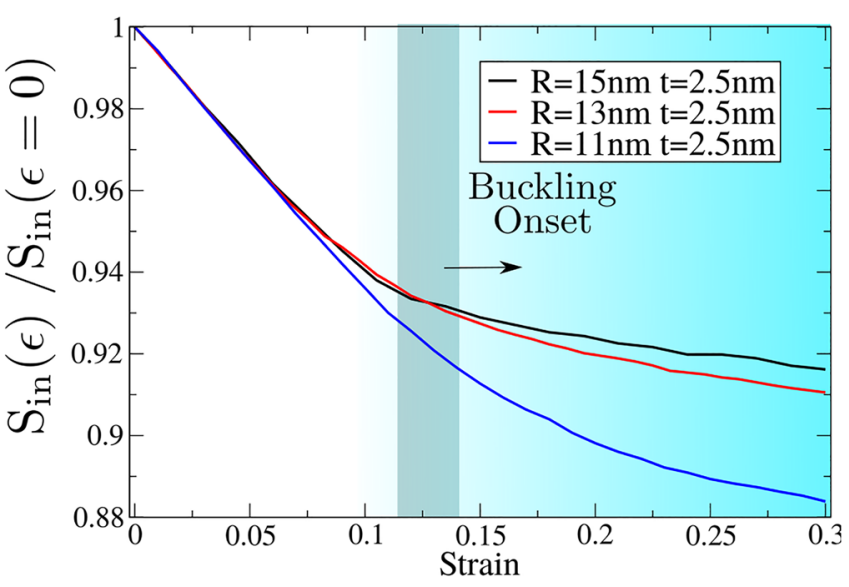

Figure 4. Role of NT diameter on the mechanical response of nc-NTs. (a) Stress-strain curve for nc-NTs of R $=11,13$, and $15 \mathrm{~nm}$ and $t=2.5 \mathrm{~nm}$. (b) Stress strain curve for nc-NTs of $\mathrm{R}=11,13$, and $15 \mathrm{~nm}$, and $t=5 \mathrm{~nm}$. (c) nc-NT buckling and coiling. Atoms are colored according to the crystalline structure obtained with a CNA analysis: green $=\mathrm{fcc}$, red $=\mathrm{hcp}$, and white $=$ other. Insets show the rotation angle of a single grain, obtained from the Polyhedral Template Matching algorithm (PTM), relative to zero strain. (d) depicts the inner surface $\left(S_{\text {in }}\right)$ fraction vs. strain, where the darker gray region delimits the buckling onset.

and volume variations of the NT inner surface. The volume inside the tube decreases linearly with strain, with a slope that is almost unchanged by buckling, as shown in Fig. S5. This is because buckling is highly localized and does not significantly affect the total pore volume. The inner surface of the NT shows this localization in Fig. S6. Inner surface area is expected to decrease due to uniaxial deformation, but increase due to buckling. The net effect, shown in Fig. 4d, is that the inner surface area continues to decrease after buckling, but with a significantly smaller slope.

Dislocation analysis of Ni NTs and NWs. Figure 5 illustrates major differences in planar defect formation of crystalline and nc structures, and their evolution. In c-NTs and c-NWs a compressive strain of approximately 0.07 is required for nucleation of the earliest planar defects. In both cases plasticity is mostly dominated by twinning (there should be a narrow strain range where stacking faults (SFs) dominate before they lead to twins). In nc systems SFs and twins already appear at zero strain since the ultra-small grain size allows the nucleation of faults during nc relaxation. Major differences arise for crystals since both structures, NTs and NWs, seem to be prone to create more planar defects as a function of applied strain. Moreover, the nc-NT and nc-NW geometries have the same behavior, even at strains close to 0.3; thus, we conclude that the formation of planar defects is dominated by the grain boundary structure and not by the tubular or wire design. In nc-NTs under tension, Rojas-Nunez revealed twin dominated fracture ${ }^{17}$; however, under compression a different deformation mechanism is observed, at least for nc systems where bending can replace the shear localization observed in the crystalline case. Here nc-NTs and nc-NWs present more SFs and twins in the concave portions of the tube, where the curvature is more relevant, than in the convex regions. 


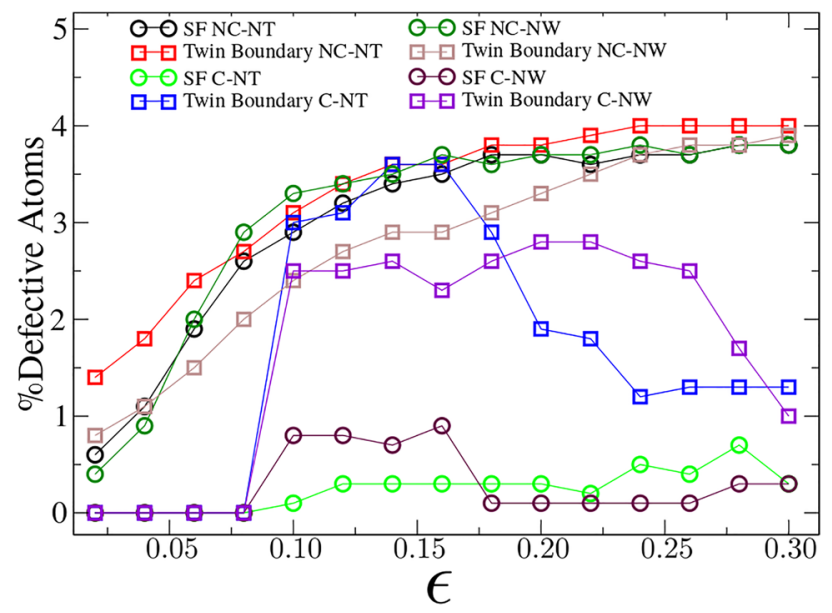

Figure 5. Planar defect analysis for NTs of $t=5 \mathrm{~nm}$ and $\mathrm{R}=11 \mathrm{~nm}$, and NWs of $\mathrm{R}=11 \mathrm{~nm}$. Defects were calculated with CAT, identifying the percentage of defective atoms in SFs and twin boundaries, for different strains.

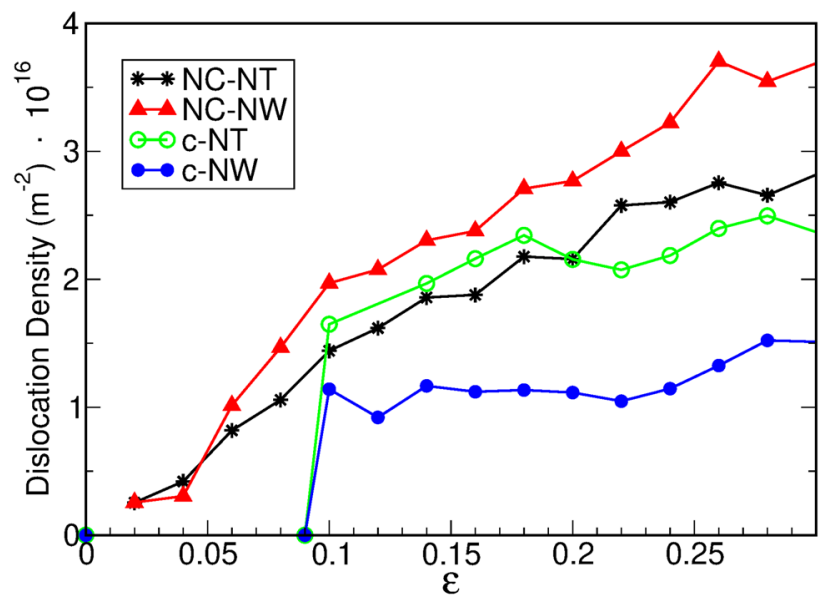

Figure 6. Dislocation density of $\mathrm{t}=5 \mathrm{~nm}$ and $\mathrm{R}=11 \mathrm{~nm}$ Ni NTs, and $\mathrm{R}=11 \mathrm{~nm}$ NWs. Both crystalline and $\mathrm{nc}$ structures are compared.

Dislocation analysis, illustrated in Fig. 6, shows that some dislocations appear at strains less than 0.02, from GB sources. Plastic strain due to dislocations is limited by the fact that dislocation lines are typically not longer than the grain diameter, and only move across a single grain. Therefore, there is a scenario of dynamical absorption and production of dislocations taking place at grain boundaries.

Dislocation counts in nc-NTs and nc-NWs show that Shockley partials $(1 / 6<112>)$ dislocations correspond to $90 \%$ of the total count, while $5 \%$ are Stair-Rod $(1 / 6<110>)$ junctions. The rest corresponds to small segments of full dislocations $(1 / 2<110>)$. In all the cases illustrated, single-crystal systems show a slower growth of dislocation density with strain, after plastic yielding. Dislocations can grow and move across the whole cross-section of the wire or NT, not only across a single grain. There is evidence of high localization of Hirth and Stair-Rod dislocations, originating from dislocation junctions, in regions confined by twin boundaries and SFs, as seen in Fig. 7. This differs from nc-systems, where dislocations tend to be distributed over the whole NT.

\section{Conclusions}

The main goal of our study of nc-NTs is motivated by its adjustable combination of geometrical parameters that mimic NW performance, but are significantly lighter and can adopt functional shapes. In this work we study, by means of molecular dynamics simulations, the uniaxial compressive response of NTs and NWs, with and without grain boundaries. We note that the inclusion of the grain boundary structure is a more realistic approach in the modeling of metallic NTs, as the ones obtained experimentally ${ }^{15-17}$.

Our conclusions can be summarized as follows: 

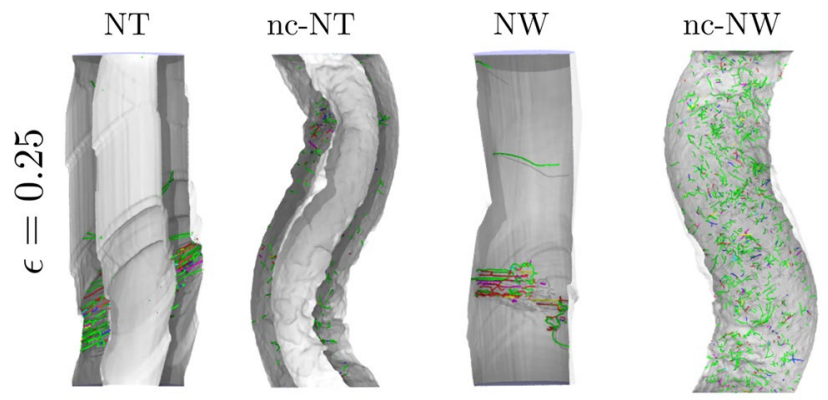

Figure 7. Dislocations for a NTs with $\mathrm{t}=5 \mathrm{~nm}$ and $\mathrm{R}=11 \mathrm{~nm} \mathrm{NTs}$, and for NWs of $\mathrm{R}=11 \mathrm{~nm} \mathrm{NWs}$ compressed to a strain of 0.25 . Only dislocations and surfaces are illustrated in the figure. We notice that, due to the presence of two surfaces, several NT dislocations are not apparent.

- For the grain sizes studied here a NT wall thickness larger than a few nm is necessary to achieve the same mechanical properties than the solid NW counterpart. Smaller thickness are dominated by surface effects and an enhanced grain boundary activity, resulting in softer structures than their thicker counterparts.

- In crystalline NTs and NWs, plasticity is always dominated by twinning and by stacking faults, led by Shockley partial dislocations in highly localized regions. In nc counterparts, GB activity also contributes significantly to plasticity, but the small grain size of the nc material leads to a uniform dislocation distribution over the whole nanostructure, avoiding any possible shear localization as observed in single crystal structures.

- The absence of only a few preferential planes allows a completely flexible behavior for nc-structures at larger strains. Crystalline shear localization is substituted by nc coiling for strains even of 0.30 . In addition, for $t=$ $2.5 \mathrm{~nm}$, plastic deformation is dominated by the NT and wall thickness buckling, both taken place simultaneously. The surface buckling is activated by the small thickness, which leads to material diffusion to the NT inner surface.

- The inner volume of the nanotubes, which could be used for transport or storage, is reduced by compression and plastic activity. Buckling can affect the rate at which this reduction occurs, and also lead to significant localized constrictions of the inner cavity. Inner surface area is also reduced by compression, and buckling diminishes its reduction rate with strain.

- Substantial differences in the mechanical properties of crystalline and nanocrystalline systems have been observed. Plastic deformation starts at larger strain for perfect crystals, due to the absence of pre-existing defects in the material ${ }^{35}$. In our case, dislocations nucleate at the surface of the c-NTs or c-NWs, as expected. On the other hand, grain boundary activity in polycrystalline systems can produce plastic deformation at small strains. At larger strains dislocations can nucleate from surfaces or from grain boundaries ${ }^{36,37}$.

- In the case of c-NWs and c-NTs, plasticity starts with surface nucleation of Shockley partial dislocations, which travel across the NT and lead to surface steps. They produce stress drops, generating a seesaw stressstrain curve, up to a strain of 0.1 . Dislocation nucleation in single crystal "perfect" NWs and NTs requires larger stress than GB activity, leading to a higher elastic limit and ultimate stress than for nc-NWs and ncNTs. The abundance of partial dislocations leads to twinning, which also reflects on surface steps and bulging in regions of high dislocation density.

- nc-NTs and nc-NWs display a different plastic behavior, without bulging. This is because a significant amount of strain is accommodated by GBs, and dislocation activity is confined by GBs which can stop or absorb impinging dislocations. The large number of grains in random orientations leads to a large number of dislocation glide-plane orientations and, therefore, the material responds as a nearly isotropic material. In this case, grain rotation in the material under high compression allows for buckling, which depends on the NT geometrical parameters, such as thickness, radius, and NT length.

Our findings are useful for the design of new light-weight and highly deformable meta-materials, taking advantage of the mass reduction and the ductile behavior of nc-NTs. Moreover, these nc-structures are softer than their defect-free crystalline counterparts. Future work will focus on grain size effects and grain boundary engineering, as possible mechanisms to modify mechanical properties.

\section{Methods}

Computational modeling of Ni nanostructures. The uniaxial compressive strain is simulated by molecular dynamics employing the LAMMPS code ${ }^{38}$. The Ni interatomic interactions are modeled using the embedded atom method ${ }^{39}$, with the parametrization proposed by Mishin et al. ${ }^{40}$, that correctly reproduces several physical quantities such as elastic constants ${ }^{41,42}$, and stacking fault energy ${ }^{43}$, amongst other properties. The nanocrystalline texture was obtained by cutting NWs and NTs from a Ni nc-bulk sample of 5 nm mean grain size. The nanocrystal bulk with specified grain sizes was built employing a Voronoi tessellation algorithm ${ }^{45}$, which provides a grain size distribution similar to the one of experiments that use atomic layer deposition techniques ${ }^{15}$. 
Cuts of the bulk sample lead to a decrease in mean grain size. To estimate the new mean grain size in NWs and NTs, grains were identified by removing GB atoms with the help of Common Neighbor Analysis (CNA), and next using cluster analysis to isolate each grain as a given cluster. Atoms in the cluster can be separated between "surface" and "interior" atoms using centrosymmetry parameter analysis. For each cluster, a center of mass (COM) is calculated, and the distance between that COM and the atoms at the periphery of the cluster gives a distribution of radii, leading to a mean grain radius. This is the same method used in the study of $\mathrm{Ni}$ NTs under tension ${ }^{17}$. As a check for the method, when applied to the bulk nc sample, we verified that it gives 5 $\mathrm{nm}$ grain size. For NTs with thickness thinner than the mean grain size, grains can have a length, along the NT axis, larger than the NT thickness; this can yield a mean grain size larger than the thickness itself. This is indeed the case for $\mathrm{t}=2.5 \mathrm{~nm}$, where we find a mean grain size of $3 \mathrm{~nm}$. Something similar has been found for hollow nanocrystalline spheres ${ }^{44}$, where the interplay between thickness and the original grain size allows for tailoring of the mechanical properties. Future studies for nanotubes might also explore this interplay.

Voronoi constructions yield grain boundary structures far from equilibrium; therefore, high energy grain boundaries have to be relaxed to avoid possible artifacts. The grain boundary structure was relaxed by means of a zero-pressure barostat coupled to a 300 K Nose-Hoover thermostat; additional details of this methodology can be found elsewhere ${ }^{45-48}$. Finally, NWs and NTs of the desired radius and thickness are carved from the nc template and relaxed again, with a velocity rescaling algorithm, at $300 \mathrm{~K}$ during $100 \mathrm{ps,} \mathrm{coupled} \mathrm{to} \mathrm{a} \mathrm{zero-pressure}$ barostat in the axial direction. This cut slightly modifies the grain size distribution, and the resulting mean grain sizes are $3.8 \mathrm{~nm}$ and $3.5 \mathrm{~nm}$ for NWs and NTs, respectively. Simulations were performed using periodic boundary conditions along the nanotube axis ( $z$-axis).

The 11,13 and $15 \mathrm{~nm}$ radius NTs, of 2.5 and $5.0 \mathrm{~nm}$ thickness, were created to investigate the role played by the NT radius. Additionally, for the smaller NTs, the wall thickness t was varied $(2.5,5.0,7.5$ and $10 \mathrm{~nm})$, to investigate the role of thickness in plasticity. A minimum shell thickness of $2.5 \mathrm{~nm}$ was adopted to avoid partial collapse and to ensure a uniform nanotube, before uniaxial compression was performed. This criterion was chosen based on Cao et al. ${ }^{21}$ findings, which show a strong surface reorientation when the NT thickness is close to $1 \mathrm{~nm}$. We note that this behavior has also been observed in other hollow structures ${ }^{49-51}$, for a specific combination of radius and thickness. In those cases, the surface stress is not compensated by the shell, originating a partial shrinkage, and the surface reorientation is assisted by Shockley partial dislocations as the dominant mechanism. As a consequence, the hollow structure reduces its radius, or roughens its surface to decrease surfaces stress.

Mechanical deformation and molecular dynamics. Uniaxial compression was applied along the $\mathrm{z}$-axis at a strain rate of $10^{8} \mathrm{~s}^{-1}$, and with a time step of $1.0 \mathrm{fs}$; the same rate was applied when unloading to zero stress. During deformation, periodic boundary condition in the $z$-axis were adopted. The system temperature was held at $300 \mathrm{~K}$ during the simulations, by means of a Nose-Hoover thermostat. The NTs and NWs were uniaxially deformed along $z$ using an NPT ensemble to keep zero pressure in $x$ and $y$. Each nanostructure was then homogeneously compressed to deformations of $30 \%$. Defect detection, visualization, and rendering were carried out with OVITO ${ }^{52}$ (https://www.ovito.org), while dislocations and planar defect identification were obtained with the Dislocation Extraction Algorithm (DXA) developed by Stukowski ${ }^{52}$, and the Crystal analysis Tool ${ }^{29}$ (CAT) (https://gitlab.com/stuko/crystal-analysis-tool).

As a measure of roughness, we computed the standard deviation $(\Delta r)$ with respect to the average NT radius, as

$$
\Delta r=\sqrt{\sum_{i=1}^{n} \frac{\left(r_{i}-\langle r\rangle\right)^{2}}{n}},
$$

where $r_{i}=\sqrt{x_{i}^{2}+y_{i}^{2}}$, and $\langle r\rangle=\frac{1}{n} \sum_{i=1}^{n} r_{i}$ is the average NT radius.

Received: 13 March 2020; Accepted: 21 October 2020

Published online: 03 December 2020

\section{References}

1. Meza, L. R., Das, S. \& Greer, J. R. Strong, lightweight, and recoverable three-dimensional ceramic nanolattices. Science 345, 1322-1326. https://doi.org/10.1126/science.1255908 (2014).

2. Zheng, X. et al. Multiscale metallic metamaterials. Nat. Mater. 15, 1100-6. https://doi.org/10.1038/nmat4694 (2016).

3. Torrents, A., Schaedler, T. A., Jacobsen, A. J., Carter, W. B. \& Valdevit, L. Characterization of nickel-based microlattice materials with structural hierarchy from the nanometer to the millimeter scale. Acta Mater. 60, 3511-3523. https://doi.org/10.1016/j.actam at.2012.03.007 (2012).

4. Valdevit, L., Godfrey, S. W., Schaedler, T. A., Jacobsen, A. J. \& Carter, W. B. Compressive strength of hollow microlattices: experimental characterization, modeling, and optimal design. J. Mater. Res. 28, 2461-2473. https://doi.org/10.1557/jmr.2013.160 (2013).

5. Schaedler, T. A., Ro, C. J., Sorensen, A. E., Yang, S. S., Carter, W. B. \& Jacobsen, A. J. Designing metallic microlattices for energy absorber applications. Adv. Eng. Mater. 16, 276-283. https://doi.org/10.1002/adem.201300206 (2014).

6. Erdeniz, D., Schaedler, T. A. \& Dunand, D. C. Deposition-based synthesis of nickel-based superalloy microlattices. Scripta Mater. 138, 28-31. https://doi.org/10.1016/j.scriptamat.2017.05.027 (2017).

7. Montemayor, L. C. \& Greer, J. R. Mechanical response of hollow metallic nanolattices: combining structural and material size effects. J. Appl. Mech. 82, 071012-071022. https://doi.org/10.1115/1.4030361 (2015).

8. Wang, W.-D., Yi, C.-L. \& Fan, K.-Q. Molecular dynamics study on temperature and strain rate dependences of mechanical tensile properties of ultrathin nickel nanowires. Trans. Nonferrous Metals Soc. China 23, 3353-3361. https://doi.org/10.1016/S1003 $-6326(13) 62875-7(2013)$.

9. Wang, B., Shi, D., Jia, J., Wang, G., Chen, X. \& Zhao, J., Elastic and plastic deformations of nickel nanowires under uniaxial compression. Physica E 30, 45-50. https://doi.org/10.1016/j.physe.2005.07.018 (2005). 
10. Park, H. S., Gall, K. \& Zimmerman, J. A. Deformation of fcc nanowires by twinning and slip. J. Mech. Phys. Solids 54, 1862-1881. https://doi.org/10.1016/j.jmps.2006.03.006 (2006).

11. Setoodeh, A. R., Attariani, H. \& Khosrownejad, M. Nickel nanowires under uniaxial loads: a molecular dynamics simulation study. Comput. Mater. Sci. 44, 378-384. https://doi.org/10.1016/j.commatsci.2008.03.035 (2008).

12. Huang, D., Zhang, Q. \& Qiao, P. Molecular dynamics evaluation of strain rate and size effects on mechanical properties of fcc nickel nanowires. Comput. Mater. Sci. 50, 903-910. https://doi.org/10.1016/j.commatsci.2010.10.028 (2011).

13. Zhou, K., Liu, B., Shao, S. \& Yao, Y. Molecular dynamics simulations of tension-compression asymmetry in nanocrystalline copper. Phys. Lett. A 381, 1163-1168. https://doi.org/10.1016/j.physleta.2017.01.027 (2017).

14. Knez, M., Nielsch, K. \& Niinistö, L. Synthesis and surface engineering of complex nanostructures by atomic layer deposition. Adv. Mater. 19, 3425-3438. https://doi.org/10.1002/adma.200700079 (2007).

15. Pereira, A., Palma, J. L., Vazquez, M., Denardin, J. C. \& Escrig, J. A soft/hard magnetic nanostructure based on multisegmented coni nanowires. Phys. Chem. Chem. Phys. 17, 5033-5038. https://doi.org/10.1039/C4CP05665E (2015).

16. Fan, H. J., Gösele, U. \& Zacharias, M. Formation of nanotubes and hollow nanoparticles based on Kirkendall and diffusion processes: a review. Small 3, 1660-1671. https://doi.org/10.1002/smll.200700382 (2007).

17. Rojas-Nunez, J., Valencia, F., Gonzalez, R. I., Bringa, E. M., Allende, S. A., Palma, J. L., Pereira, Al., Scrig, J., \& Baltazar, S. E. Mechanical performance of lightweight polycrystalline ni nanotubes. Comput. Mater. Sci. 168, 81-86 (2019).

18. Lefebvre, L. P., Banhart, J. \& Dunand, D. C. Porous metals and metallic foams: current status and recent developments. Adv. Eng. Mater. 10, 775-787. https://doi.org/10.1039/C4CP05665E (2008).

19. Trusty, T., Xu, S. \& Beyerlein, I. J. Atomistic simulations of tungsten nanotubes under uniform tensile loading. J. Appl. Phys. 126, 095105. https://doi.org/10.1039/C4CP05665E (2019).

20. Sun, M., Xiao, F. \& Deng, C. Near-ideal strength in metal nanotubes revealed by atomistic simulations. Appl. Phys. Lett. 103, 231911-231915. https://doi.org/10.1039/C4CP05665E (2013).

21. Cao, R., Deng, Y. \& Deng, C. Ultrahigh plastic flow in au nanotubes enabled by surface stress facilitated reconstruction. Acta Mater. 86, 15-22. https://doi.org/10.1039/C4CP05665E (2015).

22. Ramasubramaniam, A. \& Carter, E. A. Coupled quantum-atomistic and quantum-continuum mechanics methods in materials research. MRS Bull. 32, 913-918. https://doi.org/10.1557/mrs2007.188 (2011).

23. Monk, J. \& Farkas, D. Tension-compression asymmetry and size effects in nanocrystalline ni nanowires. Phil. Mag. 87, $2233-2244$. https://doi.org/10.1080/14786430701361404 (2007).

24. Wu, Z., Zhang, Y. W., Jhon, M. H., Gao, H. \& Srolovitz, D. J. Nanowire failure: long = brittle and short = ductile. Nano Lett. 12, 910-4. https://doi.org/10.1021/nl203980u (2012).

25. Peng, C., Zhan, Y. \& Lou, J. Size-dependent fracture mode transition in copper nanowires. Small 8, 1889-94. https://doi. org/10.1002/smll.201101911 (2012).

26. Hull, D. \& Bacon, D. J. Introduction to dislocations Vol. 37 (Elsevier, Amsterdam, 2011).

27. Ko, W.-S., Stukowski, A., Raheleh, H., Nematollahi, A., Jeon, J. B., Choi, W. S., Dehm, G., Neugebauer, J., Kirchlechner, C. \& Grabowski, B. Atomistic deformation behavior of single and twin crystalline cu nanopillars with preexisting dislocations. Acta Materialia 197(54), 68, https://doi.org/10.1016/j.actamat.2020.07.029 (2020).

28. Bringa, E., Caro, A. \& Leveugle, E. Pressure effects on grain boundary plasticity in nanophase metals. Appl. Phys. Lett. 89, 023101. https://doi.org/10.1063/1.2217188 (2006).

29. Stukowski, A. Structure identification methods for atomistic simulations of crystalline materials. Modell. Simul. Mater. Sci. Eng. 20, 045021. https://doi.org/10.1088/0965-0393/20/4/045021 (2012).

30. Diao, J., Gall, K. \& Dunn, M. L. Yield strength asymmetry in metal nanowires. Nano Lett. 4, 1863-1867. https://doi.org/10.1021/ nl0489992 (2004).

31. Jang, D. \& Greer, J. R. Size-induced weakening and grain boundary-assisted deformation in $60 \mathrm{~nm}$ grained ni nanopillars. Scripta Mater. 64, 77-80. https://doi.org/10.1016/j.scriptamat.2010.09.010 (2011).

32. Liu, Z. R. \& Zhang, R. F. Aacsd: An atomistic analyzer for crystal structure and defects. Comput. Phys. Commun. 222, 229-239. https://doi.org/10.1016/j.cpc.2017.07.026 (2018).

33. Zhang, Y. Y., Wang, C. M., Duan, W., Xiang, Y. \& Zong, Z. Assessment of continuum mechanics models in predicting buckling strains of single-walled carbon nanotubes. Nanotechnology 20, 395707. https://doi.org/10.1088/0957-4484/20/39/395707 (2009).

34. Feliciano, J., Tang, C., Zhang, Y. \& Chen, C. Aspect ratio dependent buckling mode transition in single-walled carbon nanotubes under compression. J. Appl. Phys. 109, 084323. https://doi.org/10.1063/1.3569616 (2011).

35. Alhafez, I. A., Ruestes, C. J., Bringa, E. M. \& Urbassek, H. M. Influence of pre-existing plasticity on nanoindentation-an atomistic analysis of the dislocation fields produced. J. Mech. Phys. Solids 132, 103674. https://doi.org/10.1016/j.jmps.2019.07.017 (2019).

36. Meyers, M. A., Mishra, A. \& Benson, D. J. Mechanical properties of nanocrystalline materials. Prog. Mater Sci. 51, 427-556. https ://doi.org/10.1016/j.pmatsci.2005.08.003 (2006).

37. Weertman, J. R., Farkas, D., Hemker, K., Kung, H., Mayo, H., Mitra, R., \& Van Swygenhovem, H. Structure and mechanical behavior of bulk nanocrystalline materials. MRS Bull. 24, 44-53. https://doi.org/10.1557/S088376940005154X (1999).

38. Plimpton, S. Fast parallel algorithms for short-range molecular dynamics. J. Comput. Phys. 117, 1-19. https://doi.org/10.1006/ jcph.1995.1039 (1995).

39. Daw, M. S. \& Baskes, M. I. Embedded-atom method: derivation and application to impurities, surfaces, and other defects in metals. Phys. Rev. B 29, 6443-6453. https://doi.org/10.1103/PhysRevB.29.6443 (1983).

40. Mishin, Y., Farkas, D., Mehl, M. J. \& Papaconstantopoulos, D. A. Interatomic potentials for monoatomic metals from experimental data and ab initio calculations. Phys. Rev. B 59, 3393-3407. https://doi.org/10.1103/PhysRevB.59.3393 (1999).

41. Simmons, G. \& Wang, H. Single Crystal Elastic Constants and Calculated Aggregate Properties: A Handbook 2nd edn. (The MIT Press, Cambridge, 1971).

42. Birgeneau, R. J., Cordes, J., Dolling, G. \& Woods, A. D. B. Normal modes of vibration in nickel. Phys. Rev. 136, A1359-A1365. https ://doi.org/10.1103/PhysRev.136.A1359 (1964).

43. Murr, L. E. Interfacial Phenomena in Metals and Alloys (Addison-Wesley, Boston, 1975).

44. Valencia, F. J., Pinto, B., Kiwi, M., Ruestes, C. J., Bringa, E. M., \& Rogan, J. Nanoindentation of polycrystalline pd hollow nanoparticles: grain size role. Comput. Mater. Sci. 179, 109642. https://doi.org/10.1016/j.commatsci.2020.109642 (2020).

45. Bringa, E. M., Caro, A., Wang, Y., Victoria, M., McNaney, J. M., Remington, B. A., Smith, R. F., Torralva, B. R., \& Van Swygenhoven, H. Ultrahigh strength in nanocrystalline materials under shock loading. Science 309, 1838-1841. https://doi.org/10.1126/scien ce.1116723 (2005).

46. Valencia, F. J., González, R. I., Bringa, E. M. \& Kiwi, M. Hillock formation on nanocrystalline diamond. Carbon 119, 219-224. https ://doi.org/10.1016/j.carbon.2017.04.020 (2017).

47. Valencia, F., Mella, J. D., González, R. I., Kiwi, M. \& Bringa, E. M. Confinement effects in irradiation of nanocrystalline diamond. Carbon 93, 458-464. https://doi.org/10.1016/j.carbon.2015.05.067 (2015).

48. Li, W., Wang, X., Gao, L., Lu, Y. \& Wang, W. Atomic study on tension behaviors of sub-10 nm nanopolycrystalline cu-ta alloy. Materials 12, 3913. https://doi.org/10.3390/ma12233913 (2019).

49. Valencia, F. J., Gonzalez, R. I., Tramontinca, D., Rogan, J., Valdivia, J. A., Kiwi, M., \& Bringa, E. M. Hydrogen storage in palladium hollow nanoparticles. J. Phys. Chem. C 120, 23836-23841. https://doi.org/10.1021/acs.jpcc.6b07895 (2016). 
50. Valencia, F. J. Gonzalez, R.I., Vega, H., Ruestes, C., Rogan, J., Valdivia, J. A., Bringa, E. M., \& Kiwi, M. Mechanical properties obtained by indentation of hollow pd nanoparticles. J. Phys. Chem. C 122, 25035-25042. https://doi.org/10.1021/acs.jpcc.8b072 42 (2018).

51. Reyes, P. N., Valencia, F. J., Vega, H., Ruestes, C., Rogan, J., Valdivia, J. A., \& Kiwi, M. The stability of hollow nanoparticles and the simulation temperature ramp. Inorg. Chem. Frontiers 5, 1139-1144. https://doi.org/10.1039/C7QI00822H (2018).

52. Stukowski, A. Visualization and analysis of atomistic simulation data with ovito-the open visualization tool. Modell. Simul. Mater. Sci. Eng.https://doi.org/10.1088/0965-0393/18/1/015012 (2010).

\title{
Acknowledgements
}

The authors thank the support of DICYT Project 041931BR and USA1799 Vridei 041931SB_GO, the Financiamiento Basal para Centros Científicos y Tecnológicos de Excelencia AFB180001, the Fondo Nacional de Investigaciones Científicas y Tecnológicas (FONDECYT, Chile) under Grants \#1190662, \#11190484 (FV), \#11180557 (RG), \#1200867 and \#1190727 (SA). EMB thanks support from PICT-2014-0696 (ANPCyT) and SIIP-UNCuyo Grant06/M104. This research was partially supported by the supercomputing infrastructure of the NLHPC (ECM-02) and partially supported by the supercomputing infrastructure of the HPC USACH-SEGIC (ECM-02).

\section{Author contributions}

F.V, S.B, E.B, and M.K wrote the main manuscript text. J.R and S.B performed simulations. J.R, S.B, R.G, F.V prepared manuscript figures. S.A, E.B, and M.K revised the manuscript critically for important intellectual content. All authors reviewed the manuscript.

\section{Competing interests}

The authors declare no competing interests.

\section{Additional information}

Supplementary information is available for this paper at https://doi.org/10.1038/s41598-020-76276-y.

Correspondence and requests for materials should be addressed to F.J.V.

Reprints and permissions information is available at www.nature.com/reprints.

Publisher's note Springer Nature remains neutral with regard to jurisdictional claims in published maps and institutional affiliations.

\begin{abstract}
Open Access This article is licensed under a Creative Commons Attribution 4.0 International License, which permits use, sharing, adaptation, distribution and reproduction in any medium or format, as long as you give appropriate credit to the original author(s) and the source, provide a link to the Creative Commons licence, and indicate if changes were made. The images or other third party material in this article are included in the article's Creative Commons licence, unless indicated otherwise in a credit line to the material. If material is not included in the article's Creative Commons licence and your intended use is not permitted by statutory regulation or exceeds the permitted use, you will need to obtain permission directly from the copyright holder. To view a copy of this licence, visit http://creativecommons.org/licenses/by/4.0/.
\end{abstract}

(C) The Author(s) 2020 